\title{
New Host Polymeric Framework and Related Polar Guest Co-Crystals
}

Paola Rizzo, Christophe Daniel, Anna De Girolamo Del Mauro and Gaetano Guerra

\section{Supporting information}

\section{Experimental}

Materials. The s-PS used in this study was manufactured by Dow Chemical Company under the trademark Questra 101. The ${ }^{13} \mathrm{C}$ nuclear magnetic resonance characterization showed that the content of syndiotactic triads was over $98 \%$. The weight-average molar mass obtained by gel permeation chromatography (GPC) in trichlorobenzene at $135^{\circ} \mathrm{C}$ was found to be $\mathrm{M}_{\mathrm{w}}=3.2 \times 10^{5}$ with the polydispersity index, $\mathrm{M}_{\mathrm{w}} / \mathrm{M}_{\mathrm{n}}=3.9$.

$\gamma$-form powder has been obtained by annealing at $160^{\circ} \mathrm{C}$ for 12 hours of a clathrate s-PS/ $\mathrm{CHCl}_{3}$ powder.

s-PS films, 100-120 $\mu \mathrm{m}$ thick, were obtained by extrusion of the melt with an extrusion head of $200 \mathrm{~mm} \times$ $0.5 \mathrm{~mm}$. These films were oriented by uniaxial stretching, at draw ratio $\lambda=($ initial length/final length $) \approx 3$ at constant deformation rate of $0.1 \mathrm{sec}^{-1}$, in the temperature range $105-110^{\circ} \mathrm{C}$ with a Brukner stretching machine. The stretched films are essentially amorphous and become semicrystalline by exposure for three days to $\mathrm{CS}_{2}$ vapors, thus presenting the s-PS/CS 2 clathrate phase. Uniaxially oriented nanoporous $\delta$ form films have been obtained from s-PS/ $\mathrm{CS}_{2}$ clathrate films by vacuum treatment at $40^{\circ} \mathrm{C}$ for three days. Uniaxially oriented $\gamma$-form films have been obtained from $\delta$ form films by annealing at $160^{\circ} \mathrm{C}$ for 12 hours.

The orientation of the crystalline phases of the uniaxially oriented s-PS films is characterized by high degree of parallelism of the chain axes with respect to the stretching direction (the Herman's orientation factor $f_{\mathrm{c}}$ is close to 0.95$)$.

$\delta$-form and $\gamma$-form films with (002) uniplanar orientation have been obtained by guest extraction and thermal treatment at $160^{\circ} \mathrm{C}$, respectively, of a s-PS/trichloroethylene co-crystalline films, which have been obtained by s-PS solution casting. ${ }^{10 \mathrm{c}}$ The degree of (002) uniplanar orientation, as evaluated on the basis of eqs 1 and 2 of ref.10e, for $\delta$ - and $\gamma$-form films, as well as for the derived $\varepsilon$-form films, are not far from $f_{002}=$ 0.7 .

Methods. The content of the guest molecules in the films was determined by thermogravimetric measurements (TGA).

Wide-angle X-ray diffraction patterns with nickel filtered $\mathrm{CuK} \alpha$ radiation were obtained, in reflection, with an automatic Bruker diffractometer.

Infrared spectra were obtained at a resolution of $1.0 \mathrm{~cm}^{-1}$ with a Vector 22 Bruker spectrometer equipped with deuterated triglycine sulphate (DTGS) detector and a Ge/KBr beam splitter. The frequency scale was internally calibrated to $0.01 \mathrm{~cm}^{-1}$ using a He-Ne laser. 32 scans were signal averaged to reduce the noise. Polarized infrared spectra were recorded by use of a SPECAC 12000 wire grid polarizer.

The degree of crystallinity of the $\varepsilon$ form samples as well as the related co-crystal samples, as evaluated by X-ray diffraction spectra like that one of Figure 1 is not far from $40 \%$, and similar values are obtained by the FTIR procedure described in ref.[5e]. 